
About IJMA [last updated July, $\left.1^{\text {st }}, 2021\right]$

$\checkmark$ International Journal of Medical Arts is the Official Journal of the Damietta Faculty of Medicine, AlAzhar University, Egypt

$\checkmark$ It is an International, Open Access, Double-blind, Peer-reviewed Journal

$\checkmark$ Published four times a year

$\checkmark$ The First Issue was published in July 2019

$\checkmark$ Published under the following license: Creative Commons Attribution-ShareAlike 4.0 International Public License (CC BY-SA 4.0). It had updated from the Creative Commons license [CC BY] in volume 2, Issue 4, October 2020 About IJMA

$\checkmark$ The Egyptian Knowledge Bank hosts the web site of IJMA

$\checkmark$ The Egyptian Knowledge Bank supports IJMA

$\checkmark$ IJMA follows the regulations of the International Committee of Medical Journal Editors

$\checkmark$ IJMA is indexed in the "Directory of Open Access Journals" [15 January 2021].

$\checkmark$ IJMA is indexed in JGate [29-6-2021]

$\checkmark$ IJMA is a member of the International Society of Managing and Technical Editors

$\checkmark$ Listed in "Index Copernicus", "Publons", "Academic resource index [ResearchBib]", "Electronics journal library", "Eurasian Scientific Journal Index", and "Citefactor"

$\checkmark$ IJMA introduced to the search engine [BASE] through DOAJ
Click image to reach the page

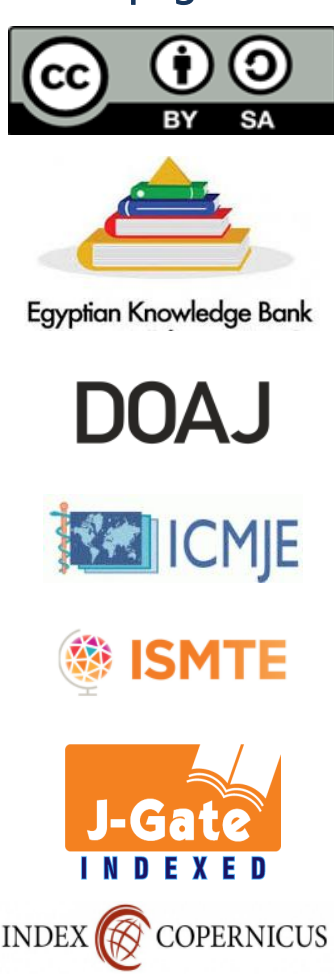

publons

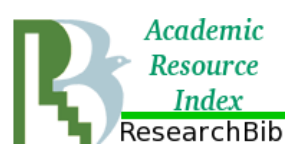

\section{EZ3 \\ .}

ESJII

CiteFactor

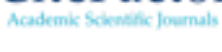

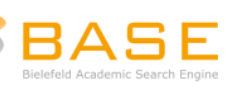




Available online at Journal Website
https://ijma.journals.ekb.eg/
Main subject [Cardiology]

Original Article

\title{
Speckle Tracking Versus Conventional Echocardiography in Patients with Symptomatic Premature Ventricular Contractions
}

\author{
Mohamed Mohamed Hal [1]; Mohamed Alshaht Omar [1]; Hammuda Abd-Al-Khalek Al-Bahnasy [1]; Mohammad Ismail \\ Al-Deftar [2]; Mohamed Moanes Mohamed [2]; Mohie AL-Dein Mohamed Samy [2] \\ 1 Department of Cardiology, Damietta Faculty of Medicine, Al-Azhar University, Egypt. \\ 2 Department of Cardiology, Faculty of Medicine, Al-Azhar University, Egypt.
}

Corresponding author: Mohamed Alshaht Omar

Email: m_omer76@yahoo.com

Submission date: April 17, 2021; Acceptance date: August 03, 2021

DOI: 10.21608/IJMA.2021.72817.1300

\section{ABSTRACT}

Background: Frequent premature ventricular complexes [PVCs] are linked to the left ventricular [LV] dysfunction. Early diagnosis is crucial for the proper treatment.

Aim of the Work: This study aimed to prove the superiority of modern diagnostic modalities as speckle tracking over the conventional echocardiography in the detection of subtle left ventricular dysfunction in symptomatic PVCs.

Patients and Methods: This study included 40 patients with frequent PVCs and 20 healthy subjects, as a comparison group. The myocardium strain in the circumferential $[\mathrm{CS}]$, radial $[\mathrm{R}]$ and longitudinal $[\mathrm{L}]$ directions were evaluated using conventional echocardiography and speckle tracking imaging.

Result: The study included 21 male and 39 females with age ranging between 22 and 56 years in the study group and between 22 and 57 years in the control group. In the case group, PVCs were more frequent [18.73 \pm 7.15$]$. The two groups have normal resting conventional echocardiography with no evidence of structural heart or systemic disease. In the study group, 25 patients have PVCs arising from the right ventricle [RV] [62.5\%] and 15 patients have PVCs arising from the LV [37.5\%]. There was no significant correlation between PVCs origin and LV global longitudinal strain [GLS], radial strain [RS] and circumferential strain [CS] [p value $=0.285,0.380$ and 0.401] respectively. There was a significant inverse correlation between the total number of PVCs [PVC burden] and LV ejection fraction [EF], GLS, RS, and CS [p value <0.001].

Conclusion: The study concluded that speckle tracking echocardiography is an important and valuable tool for early identification of impairment in the LV function in patients with frequent PVCs earlier than conventional echocardiogram.

Keywords: Left ventricle; Speckle Tracking; Echocardiography; Premature; Ventricular Complexes

This is an open-access article registered under the Creative Commons, ShareAlike 4.0 International license [CC BY-SA 4.0] [https://creativecommons.org/licenses/by-sa/4.0/legalcode.

Citation: Hal MM, Omar MA, Al-Bahnasy HA, Al-Deftar MI, Mohamed MM, Samy MM. Speckle Tracking Versus Conventional Echocardiography in Patients with Symptomatic Premature Ventricular Contractions IJMA 2020; 3[3]: July-September: 1735-1740. [DOI: 10.21608/IJMA.2021.72817.1300].

* Main subject and any subcategories have been classified according to the research topic 


\section{INTRODUCTION}

Premature ventricular contractions [PVCs] are one of the common types of arrhythmias with estimated prevalence of $6 \%$ of patients aged between 45 and 65 years old, which may lead to disturbing and potentially debilitating symptoms [e.g., chest pain, palpitation, presyncope, syncope, and heart failure]. PVCs may produce several hemodynamic effects especially on LVEF [1]. The concept of PVCsstimulated cardiomyopathy was suggested after improvement of the left ventricular [LV] systolic function in patients complained of idiopathic dilated cardiomyopathy by pharmacological suppression of PVCs [2]. Also, in cases with frequent PVCs, there was progressive worsening of LV function as measured by LVEF during follow-up [3]. Bogun et al. ${ }^{[5]}$ found a reduction of LVEF in $37 \%$ of 60 patients with idiopathic PVCs greater than 10 per hour. Patients with decreased LVEF tend to have a higher burden of PVCs ${ }^{[4]}$.

The assessment of LVEF is usually achieved by conventional echocardiography or by newly introduced speckle tracking strain imaging. Previous studies in the last five year reported the ability of speckle tracking to identify subtle changes in the heart ventricles [5]. The speckle tracking echocardiography is a quantitative ultrasound modality, used for accurate assessment of myocardial function by analysis of the speckle motions registered by the routine two-dimensional sonograms. The advantages of speckle-tracking based on its non-Doppler and angleindependency. In addition, it provides an objective quantification of myocardial deformation and LV systolic and diastolic dynamics. Additionally, it calculates strain and the strain rate offline after a satisfactory image acquisition $[6,7]$.

Speckle tracking imaging permits assessment of multidirectional LV strain in radial [RS], circumferential [CS] and longitudinal [LS] directions, and RV longitudinal strain, regardless of the angle [angle-independent] ${ }^{[5]}$.

Yao et al. [8] included 30 patients with PVCs, and concluded that speckle tracking imaging could be useful in the detection of changes in ventricular function in the PVC setting before conventional echocardiogram.

\section{THE AIM OF THE WORK}

The current study aimed to prove the superiority of modern techniques of echocardiography as speckletracking and strain and strain rate over conventional echocardiography in early detection of subtle LV systolic dysfunction in patients with symptomatic PVCs.
PATIENTS AND METHODS

The study was performed at the Cardiology Department, new Damietta University Hospital, from February 2015 to February 2017. The study included all patients with symptomatic PVCs and no evidence of structural heart disease referred to our hospital during this period of time [60 patients were eligible]. The study included all patients with symptomatic PVCs and apparently normal conventional echocardiography. The study excluded patients with recent myocardial infarction [MI], or with ECG evidence of $\mathrm{Ml}$, confirmed coronary stenosis of $>50 \%$ of vessel diameter, segmental dyskinetic regions on echocardiography, significant valvular heart disease, congenital heart disease, RV abnormalities, chronic illness, age more than 70 years and patients with atrial tachyarrhythmia.

Ethical considerations: All eligible patients signed a preprepared informed consent. The study protocol was approved by the local institutional review board [IRB] of Damietta Faculty of Medicine, Al-Azhar University.

Included subjects were submitted to through clinical examination and history taking for the assessment of symptoms due to PVC [palpitation, syncope, chest pain and exertional or resting dyspnea] and their duration. New York Heart Association [NYHA] functional class was assessed for all patients. A detailed medical history was obtained from all patients. Routine laboratory analysis including renal function tests and serum electrolytes, complete blood picture, liver function tests and thyroid profile were obtained for all patients. Resting 12-Lead ECG was performed to detect morphology and origin of PVCs, evidence of myocardial infarction, evidence of another arrhythmia. Forty-eight hours Holter monitoring was done to all patients for confirmation of the presence of frequent PVCs, exclusion of the presence of other tachyarrhythmia and calculation of PVC burden. The \%PVC was calculated as the following: 100 [number of $\mathrm{PVC} /$ numbers of total heart beats per 48 hours].

Patients underwent transthoracic echocardiography at admission using Philips IE 33, using the X 5-1 phased array sector probe [frequency range 1.5-4.3 MHz]. Images were attained when patients were in the left lateral position at the end of expiration according to the guidelines of the American Society of Echocardiography ${ }^{[8]}$ and attached to a single lead ECG. All standard measurements were gained in the parasternal long- and short-axis, apical four-chamber, twochamber and apical long axis views. LV-EF, LV-ESV and LV-EDV were calculated using the modified Simpson's rule by outlining the endocardial border at the end-diastole and end-systole in the 2-chamber and four- chamber views. End-diastolic frame was recognized as the frame after 
closure of mitral valve or the frame in largest cardiac dimension in the cardiac cycle. End-systolic frame was defined as the frame before the opening of the mitral valve or the smallest cardiac dimension in the cardiac cycle ${ }^{[8]}$.

Speckle tracking echocardiography [STE] was done for all subjects [case and control] as follow: We acquired the images using the same probe for conventional echocardiography of the Philips IE33. Digital loops with three successive cardiac cycles were acquired from apical four chambers, apical long-axis, apical two chambers and short axis at basal, mid and apical levels. Frame rates had to be $>40$ frames/second. The views had identical depth, sector width, and frame rates. We analyzed the digitally stored clips offline using commercial imaging analysis software. Speckle tracking application was started and image was opened, automatically the software showed the end-systolic frame of the cardiac cycle. In this frame, endocardial border was manually traced from its beginning to its end, then the myocardium was divided by the software into six segments with generation of segmental and global longitudinal strain, velocity and displacement curves. Clinical, Holter and echocardiographic parameters were recorded and a descriptive analysis was completed and the data were collected, verified and coded.

Statistical analysis: All quantitative variables were presented in the form of their arithmetic mean and standard deviation provided that, they were normally distributed. On the other side, categorical data were presented as frequency and percentages. Chi-square or student "t" tests were used to assess the association in categorical data and difference between two means, respectively. P-value $<0.05$ was set as the margin of significance. Pearson's correlation coefficient was calculated to estimate correlation between parameters and all analysis process had been completed using Statistical Package for Social Sciences [SPSS] version 20 for Windows [IBM $\otimes S P S S \circledR$ Inc., USA] and
Microsoft Excel 2013 for windows.

\section{RESULTS}

The study included 60 patients with no evidence of structural heart disease, 40 of them have frequent PVCs [group A], and 20 had not and assigned as a control group [group B]. They were 21 male and 39 females, with an age range between 22 and 57 years [mean \pm SD age $40.75 \pm$ 8.62 years] in group $A$, and age range from 18 and 53 years [mean \pm SD age $35.80 \pm 11.47$ years] in the control group. Of the study group [group A], 9 patients [22.5\%] were smokers versus 5 patients [25\%] were smokers in the control group. In group A, 6 patients [15\%] were diabetic versus 4 patients [20\%] in the control group. Hypertension was reported in 12 patients [30\%], and 3 patients [15\%] in the case and control groups, respectively. In the study group, all patients were suffering from frequent PVCs with PVC burden $5-30 \%$ [mean $\pm S D=18.73 \pm 7.15]$. There was no significant difference between cases and control groups [Table 1]. In the study group, 25 patients have PVCs arising from the RV [62.5\%] and 15 patients have PVCs arising from the LV [37.5\%]. The ejection fraction and different strains showed statistically significant difference between study and control groups [Table 1]. The two groups had normal resting conventional echocardiography with neither evidence of structural heart disease nor systemic disease. There was no significant correlation between PVCs origin and LV GLS, RS and CS [p value $=0.285,0.380$ and 0.401 ] respectively [Table 2 and Figure 1]. All cases in group $A$, have frequent PVCs with PVC burden ranging between 5\% to 30\% [Mean $\pm \mathrm{SD}=18.73 \pm 7.15]$. There was a significant inverse correlation between the total number of PVCs [PVC burden] and LVEF, GLS, RS and CS with $p$ value $<0.001$ [Table 3, Figures 2,3]. Using GLS for diagnosis of PVCS, the area under the curve was 0.885 , indicating a good test performance with sensitivity $75.0 \%$ and specificity of $100 \%$ at a cutoff point larger than -19.5 [Table 4, figure 4]

Table [1]: Patient characteristics

\begin{tabular}{|c|c|c|c|c|c|}
\hline \multicolumn{2}{|c|}{ Variables } & Study group $[A]$ & Control group [B] & Test & $P$ value \\
\hline \multicolumn{2}{|c|}{ Age [years] } & $40.75 \pm 8.62 ; 22-57$ & $35.80 \pm 11.46 ; 18-53$ & 1.87 & 0.07 \\
\hline \multirow[t]{2}{*}{ Sex } & Male & $14[35.0 \%]$ & $7[35.0 \%]$ & 0.001 & 1.00 \\
\hline & Female & $26[65.0 \%]$ & $13[65.0 \%]$ & & \\
\hline \multicolumn{2}{|c|}{ Smoking } & $8[22.5 \%]$ & $5[25.0 \%]$ & 0.05 & 0.82 \\
\hline \multicolumn{2}{|c|}{ DM } & $6[15.0 \%]$ & $4[20.0 \%]$ & 0.24 & 0.62 \\
\hline \multicolumn{2}{|c|}{ Hypertension } & $12[30.0 \%]$ & $4[20.0 \%]$ & 0.68 & 0.40 \\
\hline \multirow[t]{2}{*}{ Origin } & LV & $15[37.5 \%]$ & - & - & - \\
\hline & RV & $25[62.5 \%]$ & - & - & - \\
\hline \multicolumn{2}{|l|}{ LVEF } & $60.42 \pm 2.92$ & $62.9 \pm 3.43$ & 2.91 & $0.005^{*}$ \\
\hline \multirow[t]{3}{*}{ Strains } & GLS & $-16.60 \pm 3.92$ & $-22.65 \pm 2.13$ & 6.42 & $<0.001^{*}$ \\
\hline & RS & $33.35 \pm 7.24$ & $45.20 \pm 3.53$ & 6.89 & $<0.001^{*}$ \\
\hline & CS & $-16.67 \pm 6.06$ & $-22.30 \pm 5.69$ & 4.04 & $<0.001^{*}$ \\
\hline
\end{tabular}




Table [2] Speckle tracking strains in relation to origin of PVCS
\begin{tabular}{|l|c|c|c|c|}
\hline & $\mathrm{RV}[\mathrm{n}=25$ & $\mathrm{LV}[\mathrm{n}=15[$ & $t$ & $P$ \\
\hline LV_GLS & $-16.08 \pm 3.70$ & $-17.47 \pm 4.26$ & 1.085 & 0.285 \\
\hline LV_RS & $32.56 \pm 7.14$ & $34.67 \pm 7.46$ & 0.888 & 0.380 \\
\hline LV_CS & $-16.04 \pm 5.86$ & $-17.73 \pm 6.50$ & 0.850 & 0.401 \\
\hline
\end{tabular}

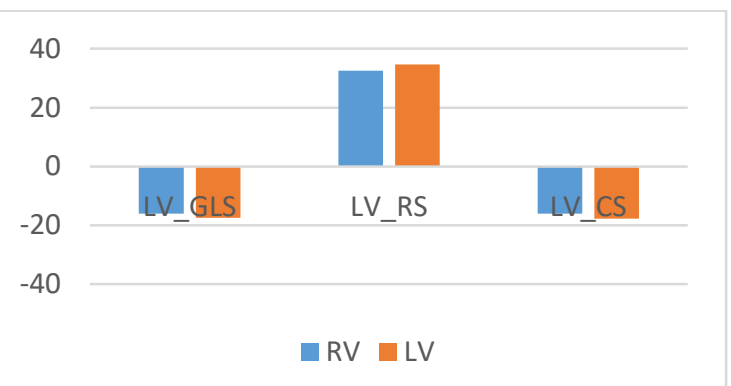

Figure [1]: Diagram showing the relation between PVCs origin and LV strains.

Table [3]: showing the correlation between PVC burden and LVEF, LV GLS, RS, CS

\begin{tabular}{|l|c|c|}
\hline \multirow{2}{*}{} & \multicolumn{2}{|c|}{ PVC burden } \\
\cline { 2 - 3 } & $\mathrm{r}$ & $\mathrm{P}$ \\
\hline LVEF & 0.882 & $<0.001^{*}$ \\
\hline LV_GLS & -0.563 & $<0.001^{*}$ \\
\hline LV_RS & 0.861 & $<0.001^{*}$ \\
\hline LV_CS & -0.875 & $<0.001^{*}$ \\
\hline
\end{tabular}

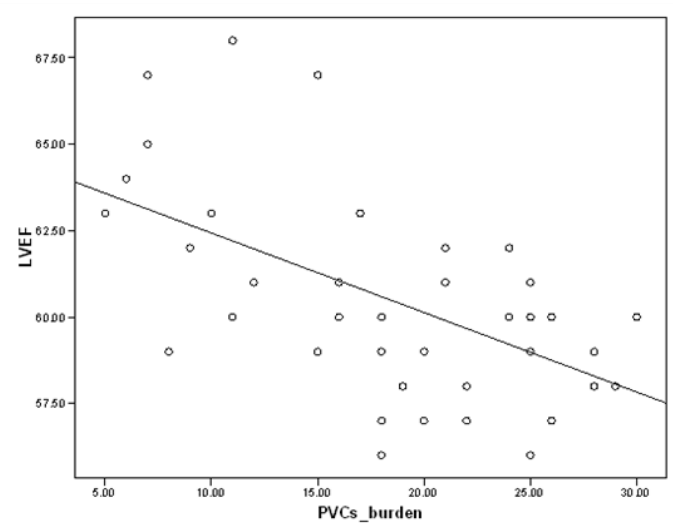

Figure [2]: Diagram showing significant inverse correlation between PVC burden and LVEF

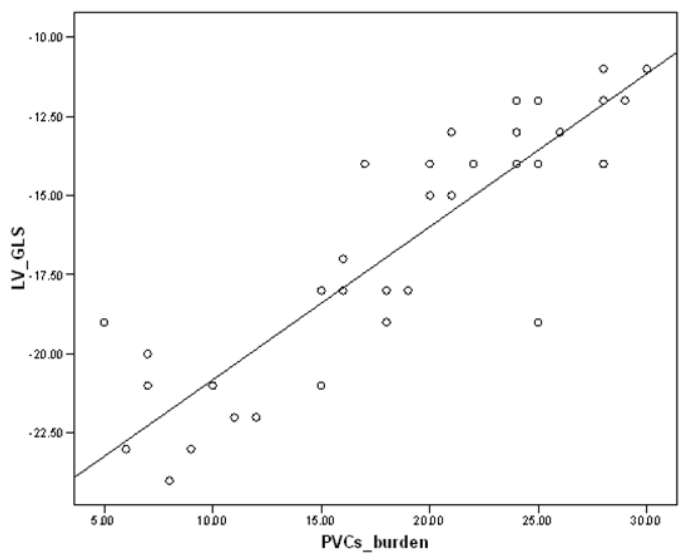

Figure [3]: Diagram showing significant inverse correlation between PVC burden and LV- GLS

\begin{tabular}{|l|c|}
\hline \multicolumn{2}{|c|}{ Table [4]: Sensitivity of GLS for diagnosis of PVCs } \\
\hline Cut off point & GLS \\
\hline Area under the curve & $>-19.5$ \\
\hline Standard error & 0.885 \\
\hline $55 \%$ confidence interval & 0.039 \\
\hline Sensitivity & $0.81-0.97$ \\
\hline Specificity & $75 \%$ \\
\hline P value & $100.0 \%$ \\
\hline
\end{tabular}

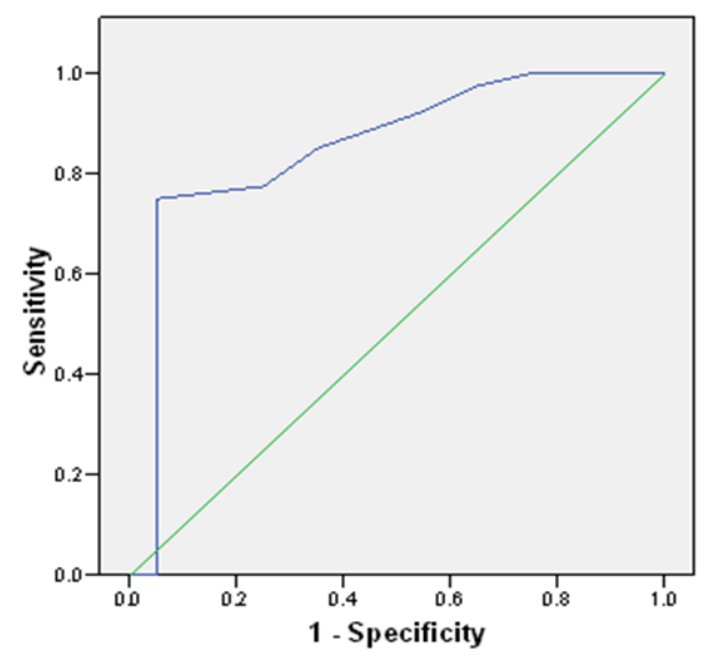

Figure [4]: Receiver operative characteristics curve for GLS in diagnosis of PVCs

\section{DISCUSSION}

PVCs are the most common arrhythmia seen by the physician and may be considered a normal finding in healthy subjects, and reported in $6 \%$ of patients aged between 45 and 65 years ${ }^{[9]}$.

Although they are always benign, in observational studies, they may be associated with a higher risk of complications [e.g., malignant arrhythmias, and sudden cardiac death] in otherwise healthy subjects ${ }^{[10,11]}$.

So, attention of researchers is paid for early recognition of LV dysfunction in patients with frequent PVCs for improvement of morbidity and mortality. The aim of this study was to prove the superiority of modern techniques of echocardiography as speckle tracking and strain and strain rate over conventional echocardiography in early detection of subtle LV systolic dysfunction in patients with symptomatic PVCs.

In the current work, there was female sex predominance [65.0\%]. The reason for a higher prevalence of women can be explained by the fact that the study population was limited to the patients with PVCs associated symptoms as females are more symptomatizing and this finding coincides 
with Wijnmaalen et al. [12] finding regarding sex distribution.

Results of the current study revealed that, there was no statistically significant differences between patients with PVCs originating from right when compared to left ventricle regarding LVEF, LV-GLS and LV-CS. Myocardial strain was reduced in all directions to a similar extent in patients with $\mathrm{LV}$ or RV origin.

These findings are comparable to Wijnmaalen et al. [12] and Del Carpio Munoz et al. ${ }^{[13]}$, regarding PVCs origin and their effect on LV systolic function.

The mean burden of PVCs in the current work was 18.73 \pm 7.15 , and there was a significant inverse correlation between PVC burden and LVEF [ $r=0.882]$, LV-GLS [ $r=-$ $0.563]$, LV-RS [ $r=0.861]$ and LV-CS $[r=-0.875]$.

These finding coincides with Niwano et al. [4], Wijnmaalen et al. [12], and Del Carpio Munoz et al. [13] finding regarding the correlation between PVC burden and LV function.

Several published studies showed that the frequency of PVCs correlates with the extent of LV dysfunction and ventricular dilation at the time of first clinical complaints. Patients with reduced LVEF had a higher PVC burden than subjects with normal function of the LV. However, there are no clear-cut points that clearly differentiate or predict when cardiomyopathy is unavoidable [14,15]. However, Niwano and coworkers ${ }^{[4]}$ used a 20.000 PVCs over 24 hours, as a cutoff point to outline the high-frequency group.

Kanei et al. ${ }^{[16]}$, on the other side, used a cutoff point of 10.000 PVCs per day.

Other studies demarcated "frequent" PVCs as $>10 \%$ of total beats rather than an absolute number of PVCs [17]; yet in some patients, a high burden of PVC may not affect the function of the LV, whereas PVC-induced cardiomyopathy can be detected in patients with low PVC burden [18]

The benign course in cases with high PVC burden is not fully understood, although one third or more of those cases could develop cardiomyopathy. One potential explanation is that, the assessment of PVC burden by Holter monitoring for 48 hours may be inadequate and could misinterpret the PVC burden ${ }^{[16]}$.

Baman et al. ${ }^{[18]}$ suggested that, 24\% PVC burden or more, yield a sensitivity of $79.0 \%$, specificity of $78.0 \%$ for differentiating LV function [preserved versus impaired]. However, patients with frequent PVCs usually had a preserved ejection fraction of the left ventricle as reported by different researchers $[3,4,17,19]$. Therefore, the PVC burden is not the only contributing or responsible factor to impaired LV systolic function, irrespective of its significance [17].

Regarding 2D speckle tracking variables of global function, there was a significant difference as regard LVGLS and CS between cases and controls. Global values of LV were significantly lower in the PVC group than in the control subjects, whereas LV function evaluated by traditional echocardiographic methods were normal. This shows that, PVCs may stimulate early LV dysfunction, which not recognized by the traditional 2D echocardiography.

This result is in accordance with Wijnmaalen et al. [12] and Yao et al. ${ }^{[8]}$.

The assessment of multidirectional strain with $2 \mathrm{D}$ speckle tracking aids the recognition of the functional deviations by exploring the myocardium mechanical properties and constitutes a more sensitive instrument to recognize the subtle ventricular dysfunction in contrast to traditional 2D echocardiography ${ }^{[20]}$.

Results of the current work are in line with Ling et al. [21] who reported non-significant differences in baseline echocardiographic variables between cases and controls. However, strain assessed variables [GLS, CS, RS] and individual segment strain was significantly reduced in the PVC group than the control group and there were strong correlations with left ventricular function assessed by left ventricular ejection fraction.

Study Limitations:

First, the study included a small number of patients. Second, the severity of symptomatic PVC was not evaluated and diastolic dysfunction was not correlated with PVC and speckle tracking parameters. Finally, the effects of various drugs such as beta-blockers and ACE inhibitors were not evaluated.

\section{Conclusion:}

The study concluded that speckle tracking echocardiography is an important and valuable tool for early detection of impairment in LV function in patients with frequent PVCs earlier than conventional echocardiogram.

\section{Financial and Non-financial Relationships and Activities of Interest}

None to be declared 


\section{REFERENCES}

1. Panizo JG, Barra S, Mellor G, Heck P, Agarwal S. Premature Ventricular Complex-induced Cardiomyopathy. Arrhythm Electrophysiol Rev. 2018 Jun; 7[2]:128-134. [DOI: 10.15420/ aer.2018.23.2].

2. Amir M, Mappangara I, Setiadji R, Zam SM. Characteristics and Prevalence of Premature Ventricular Complex: A Telemedicine Study. Cardiol Res. 2019 Oct; 10 [5]:285-292. [DOI: 10.14740/cr887].

3. You CX, Liu CF. Premature Ventricular Contractions and Cardiomyopathy. Cardiol Rev. 2019;27[6]:322-326. [DOI:10. 1097/CRD.0000000000000 262].

4. Niwano S, Wakisaka $Y$, Niwano H, Fukaya H, Kurokawa S, Kiryu M, Hatakeyama Y, Izumi T. Prognostic significance of frequent premature ventricular contractions originating from the ventricular outflow tract in patients with normal left ventricular function. Heart. 2009 Aug;95[15]:1230-7. [DOI: 10. 1136/hrt.2008.159558].

5. Lavalle C, Mariani MV, Piro A, Straito M, Severino P, Della Rocca DG, et al. Electrocardiographic features, mapping and ablation of idiopathic outflow tract ventricular arrhythmias. J Interv Card Electrophysiol. 2020 Mar; 57 [2]: 207-218. [DOI: 10.1007/s10840-019-00617-9].

6. Koester C, Ibrahim AM, Cancel M, Labedi MR. The Ubiquitous Premature Ventricular Complex. Cureus. 2020 Jan 7; 12 [1]: e6585. [DOI: 10.7759/ cureus.6585].

7. Cameli M, Mandoli GE, Sciaccaluga C, Mondillo S. More than 10 years of speckle tracking echocardiography: Still a novel technique or a definite tool for clinical practice? Echocardiography. 2019;36[5]:958-970. [DOI: 10.1111/echo.14339].

8. Yao J, Xu J, Yong YH, Cao KJ, Chen SL, Xu D. Evaluation of global and regional left ventricular systolic function in patients with frequent isolated premature ventricular complexes from the right ventricular outflow tract. Chin Med J [Engl]. 2012 Jan;125[2]:214-20. [PMID: 22340548].

9. Winkens RA, Höppener PF, Kragten JA, Verburg MP, Crebolder HF. Are premature ventricular contractions always harmless? Eur J Gen Pract. 2014 Jun; 20[2]:134-8. [DOI: 10.3109/ 13814788. 2013.859243].

10. Lin CY, Chang SL, Lin YJ, Chen YY, Lo LW, Hu YF, et al. An observational study on the effect of premature ventricular complex burden on long-term outcome. Medicine [Baltimore]. 2017; 96 [1]: e5476. [DOI: 10.1097/MD.0000000000005476].

11. Sharma E, Arunachalam K, Di M, Chu A, Maan A. PVCs, PVCInduced Cardiomyopathy, and the Role of Catheter Ablation. Crit Pathw Cardiol. 2017; 16[2]:76-80. [DOI: 10.1097/HPC. 0000000000000106].
12. Wijnmaalen AP, Delgado V, Schalij MJ, van Huls van Taxis CF, Holman ER, Bax JJ, Zeppenfeld K. Beneficial effects of catheter ablation on left ventricular and right ventricular function in patients with frequent premature ventricular contractions and preserved ejection fraction. Heart. 2010 Aug; 96 [16]: 1275-80. [DOI: 10.1136/hrt.2009.188722].

13. Del Carpio Munoz F, Syed FF, Noheria A, Cha YM, Friedman $\mathrm{PA}$, et al. Characteristics of premature ventricular complexes as correlates of reduced left ventricular systolic function: study of the burden, duration, coupling interval, morphology and site of origin of PVCs. J Cardiovasc Electrophysiol. 2011; 22 [7]: 791-8. [DOI: 10.1111/j.1540-8167.2011. 02021.x].

14. Hasdemir C, Ulucan C, Yavuzgil O, Yuksel A, Kartal Y, Simsek $\mathrm{E}$, et al. Tachycardia-induced cardiomyopathy in patients with idiopathic ventricular arrhythmias: the incidence, clinical and electrophysiologic characteristics, and the predictors. J Cardiovasc Electrophysiol. 2011 Jun; 22[6]: 663-8. [DOI: 10.1111/j.1540-8167.2010.01986. x].

15. Lee AK, Deyell MW. Premature ventricular contractioninduced cardiomyopathy. Curr Opin Cardiol. 2016 Jan; 31 [1]: 1-10. [DOI: 10.1097/ HCO.0000000000000236].

16. Kanei Y, Friedman M, Ogawa N, Hanon S, Lam P, Schweitzer $P$. Frequent premature ventricular complexes originating from the right ventricular outflow tract are associated with left ventricular dysfunction. Ann Noninvasive Electrocardiol. 2008 Jan;13[1]:81-5. [DOI: 10.1111/j.1542-474X.2007.00204. x].

17. Ban JE, Park HC, Park JS, Nagamoto Y, Choi JI, Lim HE, Park SW, Kim YH. Electrocardiographic and electrophysiological characteristics of premature ventricular complexes associated with left ventricular dysfunction in patients without structural heart disease. Europace. 2013; 15[5]:735-41. [DOI: 10.1093/ europace/eus371].

18. Baman TS, Lange DC, Ilg KJ, Gupta SK, Liu TY, Alguire C, et al. Relationship between burden of premature ventricular complexes and left ventricular function. Heart Rhythm. 2010 Jul; 7[7]:865-9. [DOI: 10.1016/j.hrthm.2010.03.036].

19. Gaita F, Giustetto C, Di Donna P, Richiardi E, Libero L, Brusin MC, Molinari G, Trevi G. Long-term follow-up of right ventricular monomorphic extra-systoles. J Am Coll Cardiol. 2001;38[2]:364-70. [DOI: 10.1016/s0735-1097[01] 01403-6].

20. Huang SJ, Orde S. From speckle tracking echocardiography to torsion: research tool today, clinical practice tomorrow. Curr Opin Crit Care. 2013;19 [3]:250-7.

21. Ling $Y$, Wan $Q$, Chen $Q$, Zhu W. Assessment of subtle cardiac dysfunction in patients with frequent PVCs by real-time threedimensional speckle tracking echocardiography. Clin Cardiol. 2017;40 [8]: 554-558. [DOI: 10.1002/clc.22697] 

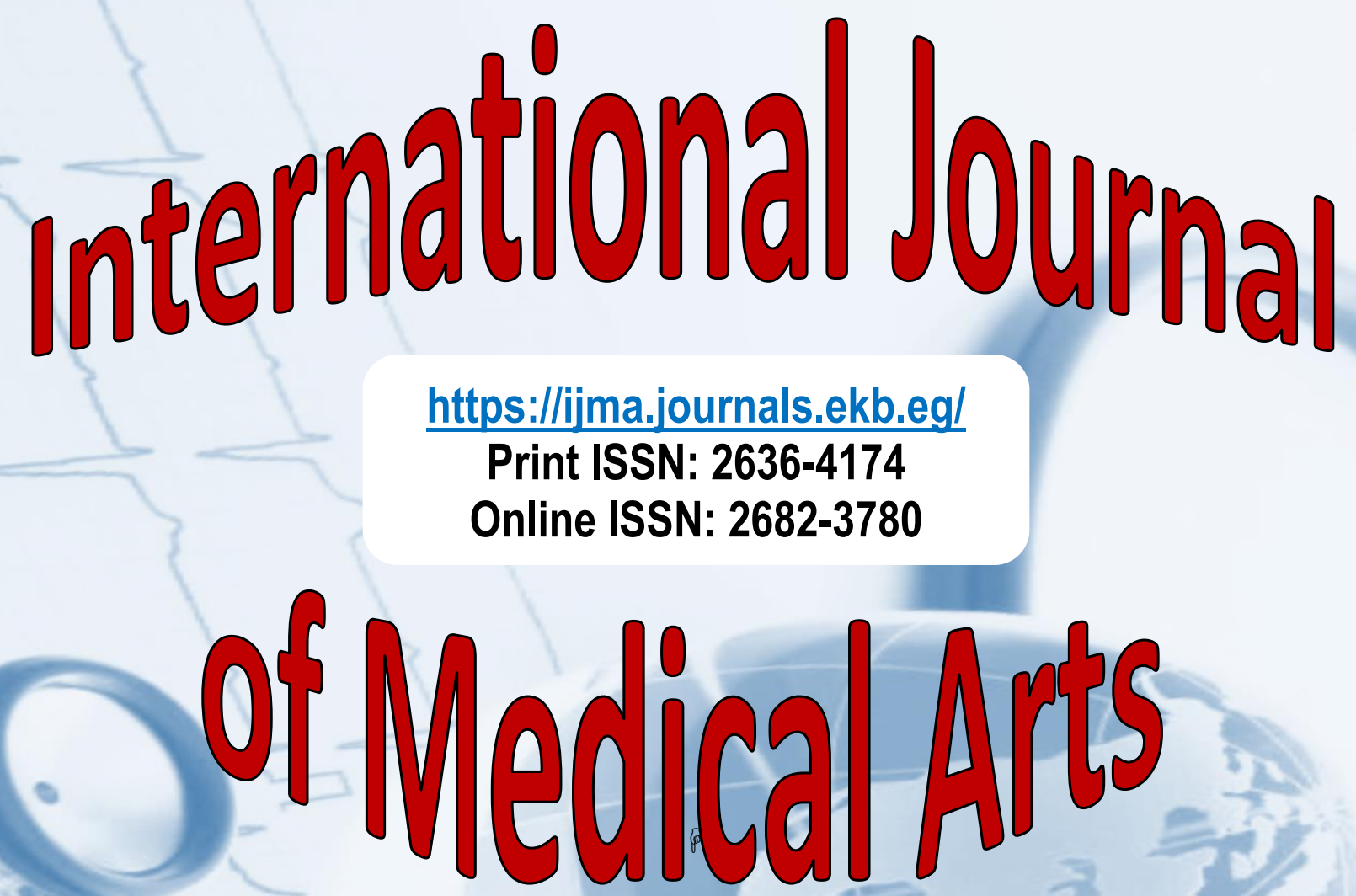